\title{
Article \\ An Introduction to the Non-Equilibrium Steady States of Maximum Entropy Spike Trains
}

\author{
Rodrigo Cofré ${ }^{1}$, Leonardo Videla ${ }^{1}$, Fernando Rosas ${ }^{2,3,4}$ \\ 1 CIMFAV, Facultad de Ingeniería, Universidad de Valparaíso, Valparaíso, Chile; \\ rodrigo.cofre@uv.cl,leonardo.videla@uv.cl \\ 2 Centre for Psychedelic Research, Department of Medicine, Imperial College London, London, UK; \\ f.rosas@imperial.ac.uk \\ 3 Centre for Complexity Science and Department of Mathematics, Imperial College London, London, UK \\ 4 Data Science Institute, Imperial College London, London, UK \\ * Correspondence: rodrigo.cofre@uv.cl
}

\begin{abstract}
Although most biological processes are characterized by a strong temporal asymmetry, several popular mathematical models neglect this issue. Maximum entropy methods provide a principled way of addressing time irreversibility, which leverages powerful results and ideas from the3literature of non-equilibrium statistical mechanics. This article provides a comprehensive overview of these issues, with a focus in the case of spike train statistics. We provide a detailed account of the5mathematical foundations and work out examples to illustrate the key concepts and results from non-equilibrium statistical mechanics.
\end{abstract}

Keywords: non-equilibrium steady states; maximum entropy principle; spike train statistics; entropy production

\section{Introduction}

Being the brain one of the most complex system within the observable universe, it is not surprising that there is still a large number of unanswered questions related to its structure and functions. With the aim of developing new ways of addressing such questions, there is an increasing consensus among neuroscientists in that interdisciplinary approaches are promising. As a prominent example of this, computational neuroscience have been greatly enriched during the last decades by tools, ideas and methods coming from statistical physics [1,2]. Moreover, these methods are recently being revisited with renewed interest due to the arrival of experimental techniques that generate huge volumes of data. In particular, neuroscientists have become progressively aware of the powerful computational techniques used by statistical physicist to analyze experimental data and large scale simulations.

When studying the firing patterns of collections of neurons, one of the most popular methods from statistical mechanics is the maximum entropy principle (MEP), which builds the least structured model that is consistent with average values measured from experimental data. These average values are usually restricted to firing rates and synchronous pairwise correlations, which gives rise to models composed by time independent and identically distributed (i.i.d) random variables, i.e. stochastic processes without temporal structure [3-5]. Needless to say, there exists strong evidence in favour of memory effects playing a major role in spike train statistics, and biological process in general [6-9]. Following this evidence, over the last years the study of complex biological systems has started to consider time-dependent processes where the past have an influence on future behavior [10-12]. The corresponding asymmetry between past and future is called the "arrow of time", which is the unique direction associated with the irreversible flow of time that is noticeable in most biological systems. 
Interestingly, the statistical physics literature has a fertile toolkit for studying time asymmetric processes [13]. First, one introduces the distinction between steady states that imply thermal equilibrium, and steady states that still carry fluxes - being called non-equilibrium steady states (NESS). Additionally, the extend to which a steady-state is not in equilibrium (i.e. the strength of it associated currents) can be quantified by the entropy-production rate [14], which is associated with the degree of time-irreversibility in the corresponding process [14]. Theoretical studies have recognized that being out-of-equilibrium is one of the distinctive properties of living systems [15-17]. Consequently, any statistical description that is consistent with the out-of-equilibrium condition of living neuronal networks should reflect some degree of time asymmetry, which can be characterized using Markov chains [11,18-22].

Despite of the potential of interdisciplinary pollination in these fascinating issues, many scientists find hard to explore these possibilities because for the major entry barriers, which include differences in jargon, conventions, and notations across the various fields. With this in mind, and with the aim to foster the collaboration among disciplines, this article provides an introduction to these topics suitable for researchers in the fields of physics or mathematics who are curious about the interesting questions and possibilities that computational neurosciences offers. The focus on this community is motivated by the growing community of mathematical physicists interested in computational neroscience.

The rest of this article is structured as follows. First, Section 2 introduce basic concepts of neural spike trains and Markov processes. Then, Section 3 introduces the notion of observable, and explore their fundamental properties. Section 4 then introduces the core ideas of MEP, proposing the formal question and exploring methods for solving it. Section 5 studies various properties of interest of MEP models, including fluction-dissipation relationships, and their entropy production. Finally, Section 6 summarizes our conclusions.

\section{Preliminary considerations}

This section introduces definitions, notations, and conventions that are used throughout the article in order to provide the necessary background to the unfamiliar reader.

\subsection{Binning and spike trains}

Consider a network of $N$ spiking neurons, where time has been binned (i.e. discretized) in such a way that each neuron can exhibit no more than one action potential within one time bin $\Delta t_{b}$. Action potentials, or "spikes", are "all-or-none" events, and hence spike data can be encoded using sequences of zeros and ones. A spiking state is denoted by $x_{t}^{k}=1$, and correspond to the event in which the $k$-th neuron spikes during the $t$-th time bin, while $x_{t}^{k}=0$ implies that it remains silent.

A spike pattern corresponds to the spike-state of all neurons at time bin $t$, denoted by $x_{t}:=\left[x_{t}^{k}\right]_{k=1}^{N}$. A spike block is a consecutive sequence of spike patterns, denoted by $x_{t, r}:=\left[\boldsymbol{x}_{s}\right]_{s=t}^{r}$ (see figure 1). While the length of the spike block $x_{t, r}$ is $r-t+1$, is also useful to consider spike blocks of infinite length starting from time $t=0$, which are denoted by $x$. Finally, in this work we consider that a spike train is an infinite sequence of spiking patterns. This assumption turn out to be useful because it allows to put our analysis in the framework of stochastic processes, and because it allows to characterize asymptotic statistical properties.

The set of all possible spike patters (or state space) corresponding to a network of $N$ neurons is denoted by $\mathbb{S}$, and the set of possible spike blocks of length $R$ corresponding to a network of $N$ neurons is denoted by $\mathbb{S}^{R}$. 

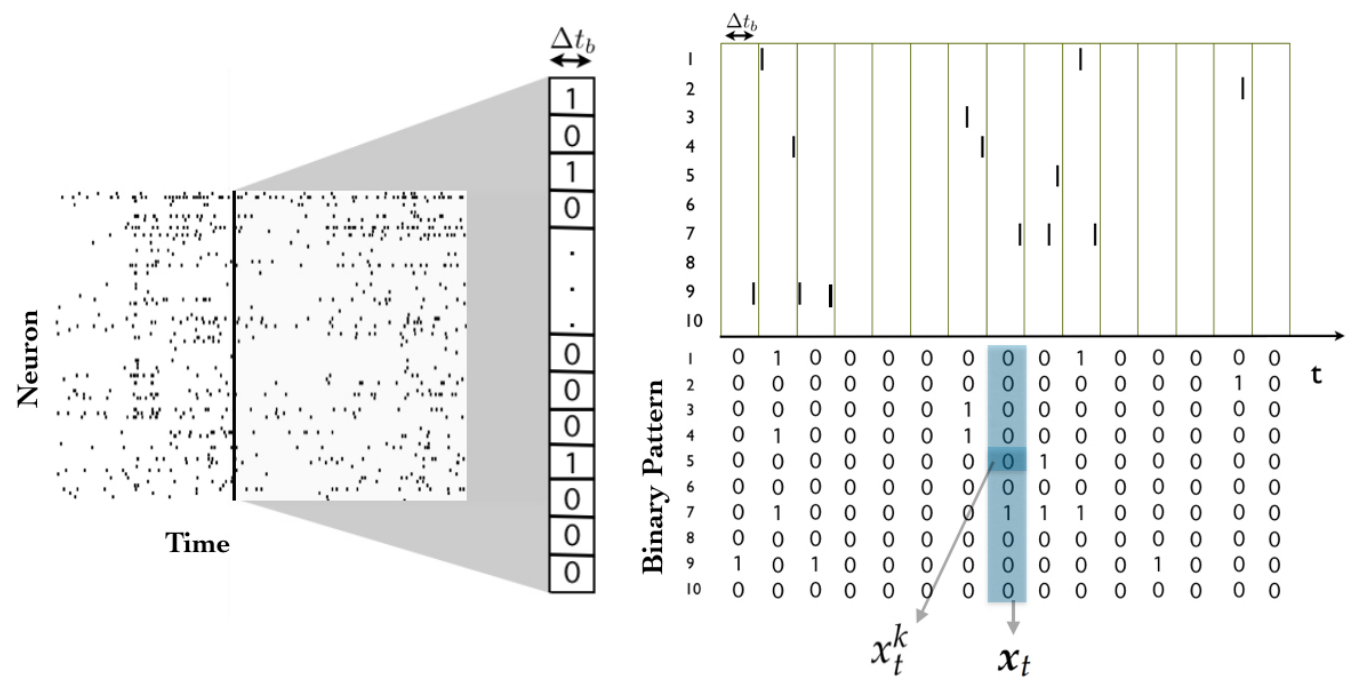

Figure 1. Illustration of a spike train, a spiking state and spike pattern. The time bin size $\Delta t_{b}$ determine the binary patterns.

\subsection{Elementary properties of Markov chains}

A stochastic process is a collection of random variables $X_{t} \in \mathbb{S}$ indexed by $t \in T$ that often refers to time. The set $\mathbb{S}$ represents the phase-space of the process; in the case of stochastic processes representing spike trains, one usually takes $\mathbb{S}=\{0,1\}^{N}$. Moreover, considering the temporal binning discussed in Section 2.1, usually $T=\mathbb{N}$, corresponding to the so-called discrete-parameter stochastic processes.

While spike trains can be characterized by stochastic processes dependent on an infinite past (non-Markovian) in mathematical models [23,24], Markov chains are particularly well-suited for modeling data sequences with not-too-strong temporal dependencies. A stochastic process $\left(X_{t}: t \in \mathbb{N}\right)$ defined on a measure space $\Omega$ is said to be a $\mathbb{P}$-Markov chain if it satisfies the Markov property (with respect to a probability measure $\mathbb{P}$ ): if, for every $t \in \mathbb{N}$ and for each sequence of states $x_{0}, x_{1}, \ldots, x_{t+1} \in$ $\mathbb{S}$, the following relationship holds:

$$
\mathbb{P}\left(X_{t+1}=x_{t+1} \mid X_{0}=x_{0}, X_{1}=x_{1}, \ldots, X_{t-1}=x_{t-1}, X_{t}=x_{t}\right)=\mathbb{P}\left(X_{t+1}=x_{t+1} \mid X_{t}=x_{t}\right) .
$$

This property is known as the Markov property, and is usually paraphrased as: the conditional distribution of the future given the current state and all past events depends exclusively on the current state of the process. It is direct to show that the Markov property is equivalent to satisfy the following condition: for every increasing sequence of indices $\left(i_{1}<i_{2}<\ldots<i_{n}\right)$ in $\mathbb{N}$, and for arbitrary states $x_{i_{1}}, x_{i_{2}}, \ldots, x_{i_{n}}$ in $\mathbb{S}$, then

$$
\mathbb{P}\left(X_{i_{n}}=x_{i_{n}} \mid X_{i_{n-1}}=x_{i_{n-1}}, \ldots, X_{i_{1}}=x_{i_{1}}\right)=\mathbb{P}\left(X_{i_{n}}=x_{i_{n}} \mid X_{i_{n-1}}=x_{i_{n-1}}\right) .
$$

To characterize the transition probabilities, define a $\mathbb{S}$-indexed stochastic matrix to be a doubly indexed array of non-negative real numbers $P=(p(i, j): i, j \in \mathbb{S})$ such that $\sum_{j \in \mathbb{S}} p(i, j)=1$ for every $i \in \mathbb{S}$. It can be shown that a Markov chain is well-defined if the following is provided:

(i) An initial probability distribution, encoded by a vector $\mu:=\left(\mu_{i}: i \in \mathbb{S}\right)$.

(ii) A collection of $\mathbb{S}$-indexed stochastic matrices $\left\{P_{t}:=\left(p_{t}(i, j)\right)_{i, j \in \mathbb{S}}: t \in \mathbb{N}\right\}$. 
Using these two elements one can build probability measures $P^{n}$ on $\mathbb{S}^{n}$ as follows,

$$
P^{n}\left(i_{0}, i_{1}, \ldots, i_{n-1}\right)=\mu\left(i_{0}\right) \prod_{j=0}^{n-2} P_{j}\left(i_{j}, i_{j+1}\right)
$$

Furthermore, the Kolmogorov extension theorem [25] guarantees the existence of a unique probability measure $\mathbb{P}_{\mu}$ on $\mathbb{S}^{\mathbb{N}}$ such that the coordinate process satisfies:

$$
\mathbb{P}_{\mu}\left(X_{0}=i_{0}, X_{1}=i_{1}, \ldots, X_{n}=i_{n}\right)=P^{n}\left(i_{0}, i_{1}, \ldots, i_{n}\right),
$$

with respect to which $\left(X_{t}: t \in T\right)$ is a Markov chain. In this case $\mathbb{P}_{\mu}$ is said to be the probability law of the Markov chain $\left(X_{t}: t \in \mathbb{N}\right)$. This notation also remarks that $\mathbb{P}_{\mu}$ is the law with initial distribution $\mu$.

\subsection{Homogeneity, ergodicity, and stationarity}

A Markov chain is said homogeneous if the transition matrices do not depend on the time parameter $t$, i.e. if there exists a $\mathbb{S}$-indexed stochastic matrix $P$ such that $P_{t}=P$ for every $t \in T$. Note that if $\left(X_{t}: t \in T\right)$ is a $\mathbb{P}$-homogeneous Markov chain, then for every $t \in T$ :

$$
\mathbb{P}\left(X_{t+1}=j \mid X_{t}=i\right)=p(i, j):=p_{i j} .
$$

s In the rest of this paper we focus exclusively on homogeneous Markov chains.

Consider now a Markov chain $\left(X_{t}: t \in T\right)$ with initial distribution $\mu$ and transition matrix $P$. Moreover, consider $p_{i j}^{(m)}$ to be the $(i, j)$-th entry of the product matrix $P^{m}=P \cdot P \cdot \ldots \cdot P$. These quantities correspond to the $m$-steps transition probabilities. Equation (2) can be generalized to

$$
\mathbb{P}\left(X_{t+m}=j \mid X_{t}=i\right)=p_{i j}^{(m)} .
$$

A stochastic matrix $P$ is said to be ergodic if there exists $k \in \mathbb{N}$ such that all the $k$-step transition probabilities are positive -i.e. there is a non-zero probability to go between any two states in $k$ steps. A homogeneous Markov chain is ergodic if it can be defined by an initial distribution $\mu$ and an ergodic matrix.

Finally, a probability distribution $\pi$ on $\mathbb{S}$ is called a stationary distribution for the Markov chain specified by $P$ if

$$
\pi P=\pi .
$$

Equivalently, $\pi$ is stationary for $P$ if $\pi$ is a left eigenvector of the transition matrix corresponding to the eigenvalue $\lambda=1$, and is a probability distribution on $\mathbb{S}$. While it is true that 1 is always an eigenvalue of $P$, it may be the case that no eigenvector associated to it can be normalized to a probability distribution. Further conditions for existence and uniqueness will be given in the next paragraph. Finally, if a $\mathbb{S}$-indexed stochastic matrix $P$ admits a stationary probability distribution $\pi$ and $\left(X_{t}: t \in \mathbb{N}\right)$ is a Markov chain with initial distribution $\pi$ and transition matrix $P$, then for every $t \in \mathbb{N}$ and $i \in \mathbb{S}$ :

$$
\mathbb{P}_{\pi}\left(X_{t}=i\right)=\pi_{i}
$$

In this case $\left(X_{t}: t \in \mathbb{N}\right)$ is said to be a stationary Markov chain, or that the Markov chain is started from stationarity.

The notion of homogeneous ergodic Markov chains is relevant in the context of spike train statistics, because of the Ergodic Theorem for finite-state Markov Chains, which state that for all finite-state, homogeneous, ergodic Markov chains $\left(X_{t}: t \geq 0\right)$ with transition matrix $P$ the following hold: 
98 (a) There exists a unique stationary distribution $\pi$ for $P$ that satisfies that $\pi_{i}>0$ for every $i \in \mathbb{S}$.

(b) For every $j \in \mathbb{S}$,

$$
\lim _{m \rightarrow+\infty} p_{i j}^{(m)}=\pi_{j}
$$

Equivalently, for every distribution $v, \lim _{t \rightarrow \infty} \mathbb{P}_{v}\left(X_{t}=j\right)=\pi_{j}$. This property guarantees the uniqueness of the maximum entropy Markov chain.

\subsection{The reversed Markov chain}

Let $\vec{P}$ be a stochastic matrix, and assume that it admits a stationary probability measure $\pi$. Assume too that $\pi_{i}>0$ for every $i \in \mathbb{S}$ (according to (a) in the Ergodic Theorem of the previous section, this is the case when $\vec{P}$ is ergodic.)

Define the $\mathbb{S}$-indexed matrix $\overleftarrow{P}$ with entries:

$$
\overleftarrow{P}_{i j}=\frac{\pi_{j}}{\pi_{i}} \vec{P}_{j i}
$$

A direct calculation shows that $\overleftarrow{P}$ is also a stochastic matrix. Moreover, if $\pi$ is stationary for $\vec{P}$, then it is for $\overleftarrow{P}$ as well.

Using the above facts, let $\mathbb{P}_{\pi}^{\rightarrow}$ and $\mathbb{P}_{\pi}^{\leftarrow}$ be the laws of two stationary Markov chains, denoted by $X_{t}$ and $Y_{t}$, whose stationary distribution is $\pi$ and transition probabilities is $\vec{P}$ and $\overleftarrow{P}$, respectively. The following holds

$$
\begin{aligned}
\mathbb{P}_{\pi}^{\overleftarrow{\pi}}\left(Y_{0}=i_{0}, Y_{1}=i_{1}, \ldots, Y_{n}=i_{n}\right) & =\pi_{i_{0}} \overleftarrow{P}_{i_{0} i_{1}} \overleftarrow{P}_{i_{1} i_{2}} \ldots \overleftarrow{P}_{i_{n-1} i_{n}} \\
& =\pi_{i_{0}} \frac{\pi_{i_{1}}}{\pi_{i_{0}}} \vec{P}_{i_{1} i_{0}} \frac{\pi_{i_{2}}}{\pi_{i_{1}}} \vec{P}_{i_{2} i_{2}} \ldots \frac{\pi_{i_{n}}}{\pi_{i_{n-1}}} \vec{P}_{i_{n} i_{n-1}} \\
& =\pi_{i_{n}} \vec{P}_{i_{n} i_{n-1}} \vec{P}_{i_{n-1} i_{n-2}} \ldots \vec{P}_{i_{1} i_{0}} \\
& \left.=\mathbb{P}_{\pi} \vec{X}_{0}=i_{n}, X_{1}=i_{n-1} \ldots, X_{n}=i_{0}\right)
\end{aligned}
$$

By virtue of this result, it is natural to call the chain $\left(Y_{t}: t \geq 0\right)$ the reversed chain associated to $\left(X_{t}: t \geq 0\right)$.

\subsection{Reversibility and detailed balance}

A transition matrix $P$ is reversible with respect to $\pi$ if the associated Markov chain started from $\pi$ has the same law as the reversed chain started from the same distribution. The reversibility of $P$ with respect to $\pi$ is equivalent to the condition of detailed balance, given by

$$
\pi_{i} P_{i j}=\pi_{j} P_{j i} \quad \forall i, j \in \mathbb{S} .
$$

Note that any probability measure $\pi$ that satisfies detailed balance with respect to $P$ is necessarily stationary, since

$$
\sum_{i \in \mathbb{S}} \pi_{i} P_{i j}=\sum_{i \in \mathbb{S}} \pi_{j} P_{j i}=\pi_{j} \sum_{i \in \mathbb{S}} P_{j i}=\pi_{j} \quad \text { for every } j \in \mathbb{S} .
$$

The converse is however not true in general: a stationary distribution may not satisfy equation (4).

Intuitively, (4) states that, in stationary state, the fluxes between each pair of states balance each other. In contrast, detailed balance is broken when there is a cycle of three or more states in the state space supporting a net probability current - even in the steady state. Detailed balance is also interpreted as "time reversibility", as one could not distinguish the steady state dynamics of the system when going forward or backward in time. Certainly, this property is not expected in stochastic processes generated by biological systems. Several disciplines use the term "equilibrium" to refer 
to long-term behaviour, i.e. what is not transient. In this article we use the term equilibrium state exclusively to refer to probability vectors that satisfy the detailed balance conditions - given in Eq. (4). Markov chains that satisfy the detailed balance condition are referred as equilibrium steady states, and conversely, steady states that do not satisfy the detailed balance conditions are referred as Non-Equilibrium Steady States (NESS)

How to characterize (finite state, homogeneous) reversible Markov chains? Following [26], consider any finite graph $\left(\mathbb{S},\left(c_{i j}\right)_{i, j \in \mathbb{S}}\right)$, with vertex set $\mathbb{S}$ and with the edge between vertices $i$ and $j$ labelled by the non-negative edge $c_{i j}=c_{j i}$. The graph can be visualized as a system of points labelled by $\mathbb{S}$, and with a line segment between points whenever the corresponding conductance is positive. Define $c_{i}=\sum_{j \in \mathbb{S}} c_{i j}$ and the $\mathbb{S}$-indexed stochastic matrix given by

$$
p_{i j}=\frac{c_{i j}}{c_{i}}
$$

Now define $C=\sum_{i \in \mathbb{S}} c_{i}$. It is straightforward to prove that $P$ is reversible with respect to the probability measure given by

$$
\pi_{i}=\frac{c_{i}}{C}
$$

and thus it is stationary for $P$. The unique Markov chain started from $\pi$ and transition matrix $P$ is called the stationary random walk on the network $\left(\mathbb{S},\left(c_{i j}\right)_{i, j \in \mathbb{S}}\right)$. Conversely, any reversible $\mathbb{S}$-valued Markov chain can be identified with the random walk on the graph with vertex set $\mathbb{S}$ and edges given by $c_{i j}=c_{j i}=\pi_{i} p_{i j}$.

\subsection{Law of large numbers for ergodic Markov chains}

The Law of Large Numbers (LLN) that applies to independent and identically distributed random variables (i.i.d.) can be extended to the realm of ergodic Markov chains. In effect, for a given ergodic Markov chain $\left(X_{t}: t \geq 0\right)$ with stationary distribution $\pi$ and transition matrix $P$, define the random variables $N_{i}^{(T)}$ equal to the number of occurrences of the state $i$ up to time $T-1$, i.e.:

$$
N_{i}^{(T)}=\sum_{t=0}^{T-1} \mathbf{1}_{\left\{X_{t}=i\right\}}
$$

where $\mathbf{1}_{\{\cdot\}}$ is an indicator function. Similarly, define the random variables $N_{i j}^{(T)}$ as the number of occurrences of the consecutive pair of states $(i, j) \in \mathbb{S}^{2}-$ in that order - up to time $T$ - 1 , i.e.:

$$
N_{i j}^{(T)}=\sum_{t=1}^{T-1} \mathbf{1}_{\left\{X_{t-1}=i, X_{t}=j\right\}}
$$

With this, the Strong law of Large Numbers for Markov chains can be stated as follows: if $\left(X_{t}: t \geq 0\right)$ is ergodic and $\pi$ is its unique stationary distribution, then

$$
\mathbb{P}_{\mu}\left(\lim _{T \rightarrow+\infty} \frac{N_{i}^{(T)}}{T}=\pi_{i}\right)=1 \quad \text { and } \mathbb{P}_{\mu}\left(\lim _{T \rightarrow+\infty} \frac{N_{i j}^{(T)}}{T}=\pi_{i} p_{i j}\right)=1,
$$

holds for any initial distribution $\mu$. The result, in turn, implies the Weak Law of Large Numbers for Markov chains, which state that, following the above notation, for every $\varepsilon>0$ and for every starting distribution $\mu$ : 


$$
\lim _{T \rightarrow+\infty} \mathbb{P}_{\mu}\left(\left|\frac{N_{i}^{(T)}}{T}-\pi_{i}\right|>\varepsilon\right)=0 \quad \text { and } \lim _{T \rightarrow+\infty} \mathbb{P}_{\mu}\left(\left|\frac{N_{i j}^{(T)}}{T}-\pi_{i} p_{i j}\right|>\varepsilon\right)=0
$$

Let's denote by $C(\mathbb{S})$ the space of real-valued functions on $\mathbb{S}$. Clearly, any function of $C(\mathbb{S})$ can be written as $f(x)=\sum_{i \in \mathbb{S}} a_{i} \mathbf{1}_{i}(x)$ for certain constants $a_{i}, i \in \mathbb{S}$. Then, the above result generalize as: for every $f \in C(\mathbb{S})$, ergodic chain $X_{t}$, and probability distribution $\mu$, the following holds:

$$
\mathbb{P}_{\mu}\left(\lim _{T \rightarrow+\infty} \frac{1}{T} \sum_{t=0}^{T-1} f\left(X_{t}\right)=\mathbb{E}_{\pi}\left(f\left(X_{0}\right)\right)\right)=1 .
$$

This corresponds to a particular form of the Birkhoff Ergodic Theorem, which is briefly outlined in the next section and is relevant to characterize spike trains of observables as from data is possible to accurately measure average values of firing rates and correlations.

For an ergodic stationary Markov chain with a state space relatively small with respect to the sample size, this theorem guarantees that from a large sample the transition probabilities and the invariant measure can be recovered. This is not the case in spike train statistics at the population level. Consider a network of $N$ neurons where sequences of spike patterns are time independent. The spike patterns can take $2^{N}$ values (state space). For $N>10$ is not possible to observe all possible states in real experimental data nor computer simulations ( 2 hours of recordings binned at 20 milliseconds produce less than $2^{19}$ spike patterns). For $N=100$ the state space is $2^{100}$, therefore the ergodic theorem is useless to estimate the transition matrix and invariant measure. Can we learn something about the statistics of spike patterns from data given that we access to a very small fraction of the state space? The maximum entropy Markov chains will give us an answer.

\section{Observables of Markov Chains and their properties}

The notion of observable plays a central role in the study of neural models. This section discusses their nature and fundamental properties.

\subsection{Observables and their empirical averages}

Suppose a spiking neuronal network of $N$ neurons is provided. Suppose too that measurements of spike patterns for $T$ time bins have been performed. The observables of such network are real-valued functions over the possible spike blocks, denoted here by $\mathbb{B}:=\mathbb{S}^{T}$. Let $C(\mathbb{B})$ be the space of such observables, i.e., the linear space of real-valued functions $f: \mathbb{B} \mapsto \mathbb{R}$. Recall the space $C(\mathbb{S})$ of observables of range 1 , discussed at the end of the above section. This space can be naturally embedded into $C(\mathbb{B})$; thus, it can be considered as a linear subspace of the latter. More generally, the space of observables of range $R$ for $R \leq T$, denoted $C\left(\mathbb{S}^{R}\right)$, is just the space of real-valued functions on $\mathbb{S}^{R}$, that we identify with its image through the natural embedding into $C(\mathbb{B})$.

We are interested in the average of observables with respect to several probability measures. If $\mu$ is a probability measure on $\mathbb{B}$ (i.e. $\mu(\omega) \geq 0$ and $\sum_{\omega \in \mathbb{B}} \mu(\omega)=1$ ) and $f$ an observable of range $R \leq T$ i.e., $f \in C\left(\mathbb{S}^{R}\right)$, we define its expectation with respect to $\mu$ as

$$
\mu(f)=\mathbb{E}_{\mu}\{f\}:=\sum_{\omega \in \mathbb{B}} f(\omega) \mu(\omega) .
$$

Since the space of blocks of length $T$ is finite, the above sum is always finite, and thus our definition makes sense for every probability measure on $\mathbb{B}$.

In the context of spike-trains, an important class of observable is made up of $\{0,1\}$-valued functions. It can be proved that any finite-range binary observable can be written as a finite sum of 
159

finite products of functions of the form $\mathbf{1}_{\left\{X_{i}^{(j)}=1\right\}}$ that represents the event that the $j$-th neuron fires during the $i$-th bin.

Consider a spike block $x_{0, T-1}$, where $T$ is the sample length. Although in general the underlying probability measure $\mu$ that governs the spiking activity is unknown, it is sometimes meaningful to use the available data to estimate the mean values of specific observables. The range of validity of this procedure is usually based on prior assumptions about the nature of the source that originates the sample. For example it can be assumed that the sample is a short piece of an infinite path that comes running from the far past, and so it can be assumed that this piece exhibits a behavior that is close to the stationary distribution. In this case, one can consider for any number $R \leq T$ the quantity:

$$
Q\left(y_{0}, y_{1}, \ldots, y_{R-1}\right)=\sum_{j=0}^{T-R} \mathbf{1}_{\left\{x_{j, j+R-1}=\left(y_{0}, y_{1}, \ldots, y_{R-1}\right)\right\}}
$$

that counts the number of appearances of the sequence $\left(y_{0}, \ldots, y_{R-1}\right)$ as a consecutive subsequence of $x_{0, T-1}$. Now, for any set $A \subseteq \mathbb{S}^{R}$, define:

$$
\mu_{x_{0, T-1}}(A):=\frac{1}{T-R+1} \sum_{y \in A} Q(y)
$$

where the measure $\mu_{x_{0, T-1}}$ is called the empirical measure on $\mathbb{S}^{R}$ from the sample $x_{0, T-1}$. If $f$ is a observable of range $R$, the empirical average value of $f$ from the sample $x_{0, T-1}$ is

$$
\mu_{x_{0, T-1}}(f)=A_{T}(f)=\frac{1}{T-R+1} \sum_{i=0}^{T-R} f\left(x_{i, R-1+i}\right)
$$

When the empirical distribution is not explicitly stated, it is customary to write $\langle f\rangle$ to denote the average of the observable $f$ with respect to this probability measure.

\subsection{Moments and cumulants}

Observables are random variables whose average values can be determined from experimental data or from the explicit representation of the underlying measure characterizing the stochastic process generating the data. Important statistical properties of random variables are encoded in the Cumulants. We will use the cumulants later in this article to characterize maximum entropy Markov chains. Let us now introduce them.

The moment of order $r$ of a real-valued random variable $X$ is given by $m_{r}=\mathbb{E}\left(X^{r}\right)$, for $r \in \mathbb{N}$ (here we freely use the notation $\mathbb{E}$ to denote the expectation with respect to a probability measure that should be inferred from the context). The moment generating function (or Laplace transform) of a random variable is defined by:

$$
M(t)=\mathbb{E}\left(e^{t X}\right)
$$

and provided it is a function of $t$ with continuous derivatives of arbitrary order at 0 , we have that:

$$
m_{r}=\left(\frac{d^{r}}{d t^{t}} M\right)_{t=0} .
$$

The cumulants $\kappa_{r}$ are the coefficients in the Taylor expansion of the cumulant generating function, defined as the logarithm of the moment generating function, namely,

$$
\ln M(t)=\sum_{r} \kappa_{r} t^{r} / r !
$$


The relationship between the moments and cumulants can be obtained by extracting coefficients from the expansion, i.e.

$$
\kappa_{r}=\left(\frac{d^{r}}{d t^{r}} \ln (M(t))\right)_{t=0}
$$

which yields the first values:

$$
\begin{aligned}
& \kappa_{1}=m_{1}, \\
& \kappa_{2}=m_{2}-m_{1}^{2}, \\
& \kappa_{3}=m_{3}-3 m_{2} m_{1}+2 m_{1}^{3}, \\
& \kappa_{4}=m_{4}-4 m_{3} m_{1}-3 m_{2}^{2}+12 m_{2} m_{1}^{2}-6 m_{1}^{4},
\end{aligned}
$$

and so on. In particular, $\kappa_{1}$ is the mean of $f, \kappa_{2}$ is the variance, $\kappa_{3}$ the skewness and $\kappa_{4}$ the kurtosis.

\subsection{Observables and ergodicity}

Let $\theta: \Omega \mapsto \Omega$ be the shift operator that acts on a sequence $\omega \in \Omega$ as:

$$
(\theta(\omega))_{i}=\omega_{i+1},
$$

i.e., $\theta$ shifts the sequence one position to the left: through its action, we see the first coordinate of $\omega$ at the 0 -th coordinate of $\theta(\omega)$, etc. Now, assume that the Markov chain $\left(X_{t}: t \geq 0\right)$ is ergodic. Let $\pi$ be its unique stationary probability distribution. The Birkhoff Ergodic Theorem states that under the above assumptions, for every $f \in C(\mathbb{B})$ :

$$
\mathbb{P}_{\mu}\left(\lim _{N \rightarrow+\infty} \frac{1}{N} \sum_{n=0}^{N-1} f \circ \theta^{n}=\mathbb{E}_{\pi}(f)\right)=1,
$$

for every initial measure $\mu$. This equation means that under the ergodic hypothesis, the temporal averages converge to the spatial averages. The importance of this fundamental result cannot be overestimated, since this is the ultimate reason that support our confidence in the practice of regarding averages of (hopefully) large samples as faithful approximations of the true values of the expectations of the observables.

\subsection{Central limit theorem for observables}

Assume that one can access arbitrarily large spike data sequences. Consider $t \in \mathbb{N}$ and let $x_{0, t-1}$ be the spike-block of length $t$. Also, let $f$ be an arbitrary observable of fixed range $R$. The asymptotic properties of $A_{t}(f)$ are established in the following context: the finite sample is drawn from an ergodic Markov chain, i.e., $x \sim \mathbb{P}_{v}$, where $\mathbb{P}_{v}$ is the Markov probability measure of an ergodic chain $\left(X_{t}: t \geq 0\right)$ started from an arbitrary initial distribution. Let $\pi$ be the unique stationary measure for the Markov chain. Observe that by virtue of the ergodic assumption, it is guaranteed that the empirical averages become statistically more accurate as the sampling size grows, i.e.,

$$
\mathbb{P}_{v}\left(A_{t}(f) \rightarrow \mathbb{E}_{\pi}\{f\}\right)=1 .
$$

for any starting condition $v$. However, the above result does not clarifies the rate at which the accuracy improves. To approach this question, we can rely upon the important central limit theorem (CLT) for ergodic Markov chains (for datails see [27]). 
Theorem 1 (Central limit theorem for ergodic Markov chains). Under the above assumptions, and keeping notation, define:

$$
\sigma=\sqrt{\left(\mathbb { E } _ { \pi } \left(\left(f\left(X_{0}, \ldots, X_{R-1}\right)-\mathbb{E}_{\pi}\left(f\left(X_{0}, \ldots, X_{R-1}\right)\right)^{2}\right)\right.\right.} .
$$

Let $L_{t}$ be the law of the random variable $\frac{\sqrt{t}}{\sigma}\left[A_{t}(f)-\mathbb{E}_{\pi}\{f\}\right]$ under the measure $\mathbb{P}_{v}$ of an ergodic Markov chain started from an arbitrary distribution. Let $L$ be the law of a standard normal random variable. Then $L_{t} \rightarrow L$ in the sense of weak convergence of convergence in distribution. This is usually written as:

$$
\mathbb{P}_{v}\left\{\frac{\sqrt{t}}{\sigma}\left[A_{t}(f)-\mathbb{E}_{\pi}\{f\}\right] \leq x\right\} \rightarrow \frac{1}{\sqrt{2 \pi} \sigma} \int_{-\infty}^{x} e^{-\frac{s^{2}}{2 \sigma}} d s,
$$

This theorem implies that "typical" fluctuations of $A_{t}(f)$ around its long term average $\mathbb{E}_{\pi}\{f\}$ are of the order of $\sigma / \sqrt{t}$.

\subsection{Large deviations of average values of observables}

Although the CLT for ergodic Markov chains is accurate in describing typical fluctuations around the mean value, it does not say much about the likelihood of large fluctuations. While it is clear that the probability of large fluctuations of average values vanish as the sample size increases, it is sometimes valuable to characterize the decrease rate. That is what the large deviation principle (LDP) does.

The empirical average $A_{t}(f)$ satisfies a large deviation principle (LDP) with rate function $I_{f}$, defined as

$$
I_{f}(s):=-\lim _{t \rightarrow \infty} \frac{1}{t} \log p\left\{A_{t}(f)>s\right\},
$$

Theorem 2 (Gärtner-Ellis theorem). If $\lambda_{f}$ is differentiable, then the average $A_{t}(f)$ satisfies a LDP with rate function given by the Legendre transform of $\lambda_{f}$, that is

$$
I_{f}(s)=\max _{k \in \mathbb{R}}\left\{k s-\lambda_{f}(k)\right\} .
$$

Thus one can study the large deviations of empirical averages $A_{t}(f)$ first computing their SCGF from the selected model and then finding their Legendre transform. One of the most useful applications of the LDP is to estimate the likelihood that $A_{t}(f)$ take a value far from its expected value. Let us assume that $I_{f}(s)$ is a positive differentiable convex function (A classical result in LDP states that $I_{f}(s)$ is a convex function if $\lambda_{f}(k)$ is differentiable [30]. For a discussion about the differentiability of $\lambda_{f}(k)$ see [31].) Then, because of the properties of convex functions $I_{f}(s)$ has a unique global minimum. Denoting this minimum by $s^{*}$, it follows from the differentiability of $I_{f}(s)$ that $I_{f}\left(s^{*}\right)=0$. Using 
properties of the Legendre transform $s^{*}=\lambda_{f}^{\prime}(0)=\mathbb{E}_{p}\{f\}$. This is the LLN, i.e., $A_{t}(f)$ concentrate around $s^{*}$. Consider a value $s \neq s^{*}$ and assume that $I_{f}(s)$ admits a Taylor series around $s^{*}$ given by

$$
I_{f}(s)=I_{f}\left(s^{*}\right)+I_{f}^{\prime}\left(s^{*}\right)\left(s-s^{*}\right)+\frac{I_{f}^{\prime \prime}\left(s^{*}\right)\left(s-s^{*}\right)^{2}}{2}+O\left(s-s^{*}\right)^{3} .
$$

Since $s^{*}$ is zero and a minimum of $I(s)$, the first two terms in this series vanish. As $I(s)$ is convex function $I^{\prime \prime}(s)>0$. For large values of $t$, we obtain from (6)

$$
\begin{aligned}
p\left\{A_{t}(f)>s\right\} & \approx e^{-t I_{f}(s)} \\
& \approx e^{-t\left(\frac{I_{f}^{\prime \prime}\left(s^{*}\right)\left(s-s^{*}\right)^{2}}{2}\right)},
\end{aligned}
$$

so the "small deviations" (we are using Taylor expansion) of $A_{t}(f)$ around $s^{*}$ are Gaussian-distributed (in equation (9) $\left.1 / I_{f}^{\prime \prime}\left(s^{*}\right)=\lambda_{f}^{\prime \prime}(0)=\sigma^{2}\right)$. In this sense, large deviation theory can be seen as an extension of the CLT because it characterize not only the small deviations around $s^{*}$ but also about large deviations (not Gaussian) of $A_{t}(f)$.

\section{Building maximum entropy temporal models}

This section presents the main concepts behind the construction of maximum entropy models for temporal data. In the sequel, Subsection 4.1 introduces the concept of entropy, and then Subsection 4.2 formulates the problem of maximizing the entropy rate. Methods for solving this problem are discussed in Subsection 4.3, which are then illustrated in an example presented in Subsection 4.4.

\subsection{The entropy rate of a temporal model}

\subsubsection{Basic definitions}

In order to give mathematical meaning to the rather vague notion of uncertainty, a natural approach is to employ the well-established notion of Shannon entropy. For any probability measure $p$ defined over the state space $E$ (not necessarily $\mathbb{S}$ ), the Shannon entropy of $p$ is given by

$$
S[p]:=-\sum_{x \in E} p(x) \log p(x)
$$

Please note that this definition can be used for measures on the spaces of infinite sequences $E^{\mathbb{N}}$. However, as in most cases of interest the value saturates in infinite, this definition is not really useful for studying such models. A better notion is given by the entropy rate, which plays a crucial role in the rest of this article.

Definition (entropy rate): Let $\mu$ be a probability measure on the space of sequences $\mathbb{S}^{\mathbb{N}}$. For $n \geq 1$ let $\mu_{n}$ be the probability measure induced by $\mu$ on the initial $n$ coordinates, i.e., $\mu_{n}$ is the probability distribution on $E^{n}$ given by:

$$
\mu_{n}\left(x_{0}, x_{1}, \ldots, x_{n}-1\right)=\mu\left(\omega \in \mathbb{S}^{\mathbb{N}}: X_{i}=x_{i} \text { for } i=0,1, \ldots, n-1\right) .
$$

The entropy rate of the measure $\mu$ is defined by:

$$
\mathcal{S}[\mu]=\lim _{n \rightarrow \infty} \frac{1}{n} S\left[\mu_{n}\right] .
$$


The above definition applies to any probability distribution on the space of sequences. Intuitively, the entropy rate correspond to the entropy per time unit, and represents how much "uncertainty" is created by the process as time moves forward.

\subsubsection{The entropy rate of I.I.D. and Markov models}

Let us consider first a null model of spike activity, where there is complete statistical independence between two consecutive spike patters. For this, first recall that $\mathbb{S}=\{0,1\}^{N}$, where $N$ is the fixed number of neurons. Without loss of generality, we can enumerate the elements of $\mathbb{S}$ as $s_{1}, s_{2}, \ldots, s_{2^{N}}$. Let $v=\left(v_{1}, v_{2}, \ldots, v_{2^{N}}\right)$ be a probability measure on $\mathbb{S}$ such that:

$$
v\left(s_{k}\right)=v_{k}
$$

For a $T$-block $x=\left(x_{0}, x_{1}, \ldots, x_{T-1}\right) \in \mathbb{S}^{T}$ and for every $s \in \mathbb{S}$, we set:

$$
N_{s}^{T}(x)=\sum_{i=0}^{T-1} \mathbf{1}_{\left\{x_{i}=s\right\}}
$$

On the space of infinite spike trains $\mathbb{S}^{\mathbb{N}}$ we consider the probability $\mu=v^{\otimes \mathbb{N}}$, i.e., the product measure on the space of spike trains. Observe that the induced measure is given by:

$$
\mu_{n}\left(x_{0}, x_{1}, \ldots, x_{T-1}\right)=\prod_{k=1}^{2^{N}} v_{k}^{N_{s}^{t}\left(x_{0}, \ldots, x_{T-1}\right)}
$$

With this, a straightforward calculation shows that

$$
\mathcal{S}[\mu]=S[v]=-\sum_{k=1}^{2^{N}} v_{k} \ln \left(v_{k}\right)
$$

and in this case we observe that the entropy rate is equal to the entropy of the probability distribution induced by each coordinate map.

A reasonable next step in the hierarchy of models is to weaken the independence hypothesis and assume instead that the spike activity keeps some bounded memory of the past. For this, following the considerations of Section 2, let us consider an ergodic discrete Markov chain with transition matrix $P$ and invariant distribution $\pi$ taking values in $\mathbb{S}$. Let $\mu=\mu(P, \pi)$ the measure induced by this chain on the space $\mathbb{S}^{\mathbb{N}}$. Observe that, with the above notation:

$$
\mu_{n}\left(x_{0}, x_{1}, \ldots, x_{n-1}\right)=\pi_{x_{0}} \prod_{j=1}^{n-1} P_{x_{j-1} x_{j}}
$$

A direct computation shows that

$$
\begin{aligned}
S\left[\mu_{1}\right] & =-\sum_{\left(x_{0}, x_{1}\right) \in \mathbb{S}^{2}} \pi_{x_{0}} P_{x_{0} x_{1}} \ln \left(\pi_{x_{0}} P_{x_{0} x_{1}}\right) \\
& =-\sum_{x \in \mathbb{S}} \pi_{x} \ln \left(\pi_{x}\right)-\sum_{\left(x_{0}, x_{1}\right) \in \mathbb{S}^{2}} \pi_{x_{0}} P_{x_{0} x_{1}} \ln \left(P_{x_{0} x_{1}}\right)
\end{aligned}
$$

and induction shows that:

$$
S\left[\mu_{n}\right]=-\sum_{x \in \mathbb{S}} \pi_{x} \ln \left(\pi_{x}\right)-n \sum_{\left(x_{0}, x_{1}\right) \in \mathbb{S}^{2}} \pi_{x_{0}} P_{x_{0} x_{1}} \ln \left(P_{x_{0} x_{1}}\right) .
$$


Thus dividing by $n$ and taking the limit in equation (10), one finds that

$$
\mathcal{S}[\mu]=-\sum_{\left(x_{0}, x_{1}\right) \in \mathbb{S}^{2}} \pi_{x_{0}} P_{x_{0} x_{1}} \ln \left(P_{x_{0} x_{1}}\right) .
$$

\subsection{Entropy rate maximization under constraints}

Now we introduce the central problem of this article. Assume we have empirical data from spiking activity. Consider the empirical averages of $K$ observables, $\left\langle f_{k}\right\rangle$, for $f_{k}, k=1, \ldots, K$. We need to characterize the Markov chains that are consistent with these average values. Except for trivial and uninteresting situations, there is no finite set of empirical averages that determines uniquely a distribution $\mu$ on $\mathbb{S}^{\mathbb{N}}$ that fits the averages, in the sense that

$$
\mu\left(f_{k}\right)=\left\langle f_{k}\right\rangle \text { for } k=1, \ldots, K .
$$

Consequently, we need to impose further restrictions in order to guarantee uniqueness. A useful and meaningful approach is the so-called Maximum Entropy Markov Chain model (MEMC), which fit the unique probability measure $\mu$ among all the stationary Markov measures $v$ on $\mathbb{S}^{\mathbb{N}}$ that match the expected values of a given set of observables and that maximizes the entropy rate. Mathematically is written in the following form:

$$
\begin{array}{ll}
\max _{v \in \mathcal{M}_{\text {inv }}} & \mathcal{S}[v] \\
\text { subject to } & v\left(f_{k}\right)=\left\langle f_{k}\right\rangle_{e}=C_{k}, \quad \forall k \in\{1, \ldots, K\},
\end{array}
$$

where $\mathcal{M}_{i n v}$ is a shorthand for the sets of stationary Markov measures on $\mathbb{S}^{\mathbb{N}}$. Formally:

$$
\mathcal{M}_{\text {inv }}:=\{(\pi, P): \pi \text { is a probability on } \mathbb{S}, P \text { is stochastic, } \pi P=\pi\} .
$$

\subsection{Solving the optimization problem}

We now discuss techniques for finding models that maximize the entropy rate.

\subsubsection{Lagrange multipliers and the variational principle}

To solve the above optimization problem, let us introduce the set of Lagrange multipliers $h_{k} \in \mathbb{R}$ and an energy function $\mathcal{H}=\sum_{k=1}^{K} h_{k} f_{k}$, which is a linear combination of the chosen observables. Next, we study the following unconstrained problem, which is a particular case of the so-called variational principle of the thermodynamic formalism [32]:

$$
\mathcal{F}[\mathcal{H}]=\sup _{v \in \mathcal{M}_{\text {inv }}}\{\mathcal{S}[v]+v(\mathcal{H})\}=\mathcal{S}[\mu]+\mu(\mathcal{H}),
$$

where $\mathcal{F}[\mathcal{H}]$ is called the free energy and $v(\mathcal{H})=\sum_{k=1}^{K} h_{k} v\left(f_{k}\right)$ is the average value of $\mathcal{H}$ with respect to the measure $v$. The following holds:

$$
\frac{\partial \mathcal{F}\left[\mathcal{H}_{h}\right]}{\partial h_{k}}=\mathbb{E}_{p}\left\{f_{k}\right\}=C_{k}, \quad \forall k \in\{1, \ldots, K\},
$$

where $\mathbb{E}_{p}\{f\}$ is the average of $f_{k}$ with respect to $p$ (maximum entropy measure), which is equal (by restriction) to the average value of $f_{k}$ with respect to the empirical measure from the data.

The maximum-entropy (ME) principle [33] has been successfully applied to spike data from the cortex and the retina $[3,9,11,12,34,35]$. The approach start fixing the set of constraints determined by the empirical average of observables measured from spiking data. Maximizing the entropy, given those constraints, provides a unique probability distribution. The choice of constraints determines the statistical model. The approach of Lagrange multipliers may not be practical when trying to 
fit a MEMC. In the next section we introduce an alternative based optimization based on spectral properties.

\subsubsection{Transfer matrix method}

Let $A$ be a adjacency matrix i.e., a $\{0,1\}$-valued square matrix with rows and columns indexed by the elements of $\mathbb{S}$. If there exists a $n \geq 0$ such that

$$
A_{i j}^{n}>0
$$

for every $i, j \in \mathbb{S}$, we say that $A$ is primitive. The next well-known theorem of Linear Algebra is fundamental [36] for the uniqueness of the MEMC.

Theorem 3 (Perron-Frobenius theorem). Let $A$ be a primitive matrix. Then,

- There is a positive maximal eigenvalue $\rho>0$ such that all other eigenvalues satisfy $\left|\rho^{\prime}\right|<\rho$ Moreover $\rho$ is simple;

- There are positive left- and right-eigenvectors $u=\left(u_{1}, \ldots, u_{k}\right), v=\left(v_{1}, \ldots, v_{k}\right)$ s.t. $u A=\rho u, A v=$ $\rho v$.

Apply the above theorem to a primitive matrix $A$, and define:

$$
P_{i j}=\frac{A_{i j} v_{j}}{\rho v_{i}} ; \quad \pi_{i}=\frac{u_{i} v_{i}}{\langle u, v\rangle},
$$

where $\langle u, v\rangle$ is the standard inner product in $\mathbb{R}^{2^{N}}$ (we refer the reader to [36] for details). The matrix $P$ built above is stochastic. Moreover, $\pi$ is its unique stationary measure. We define the Parry measure to be the Markov measure

$$
\mu\left(i_{0}, i_{1}, \ldots, i_{n}\right)=\pi_{i_{0}} P_{i_{0} i_{1}}, \ldots, P_{i_{n 1} i_{n}} .
$$

The Parry measure is the unique measure of maximal entropy consistent with the adjacency matrix $A$.

Now consider a more general and useful case for our purposes. Consider constraints given by a set of empirical averages of observables, as explained in the previous section. We assume that the observables chosen have a finite maximum range $R$. From these observables the energy function $\mathcal{H}$ of finite range $R$ is built as a linear combination of these observables. Using this energy function we build a matrix denoted by $\mathcal{L}_{\mathcal{H}}$, so that for every $y, w \in \mathbb{S}^{R}$ its entries are given as follows:

$$
\mathcal{L}_{\mathcal{H}}(y, w)= \begin{cases}e^{\mathcal{H}\left(y_{1} w_{1, R-1}\right)} & \text { if } y_{1, R-1}=w_{0, R-2} \\ 0, & \text { otherwise. }\end{cases}
$$

where $y_{1} w_{R-1}$ is the block obtained by concatenation of $y_{1}$ and $w_{1, R-1}$. For range-one observables, the above matrix is defined as $\mathcal{L}_{\mathcal{H}}(y, w)=e^{\mathcal{H}(y)}$. Assuming $\mathcal{H}>-\infty$, the elements of the matrix $\mathcal{L}_{\mathcal{H}}$ are non-negative, and Furthermore in every non trivial case, the matrix is primitive and satisfies the Perron-Frobenius theorem [36]. Denote by $\rho$ the largest eigenvalue of $\mathcal{L}_{\mathcal{H}}$, which is an eigenvalue of multiplicity one and strictly larger in modulus than the rest of the eigenvalues [36]. Just as above, we denote by $\mathbf{u}$ and $\mathbf{v}$ the left and right eigenvectors of $\mathcal{L}_{\mathcal{H}}$ corresponding to the eigenvalue $\rho$. Notice that $u_{i}>0$ and $v_{i}>0$, for all $i \in \mathbb{S}$. The free energy associated to a transfer matrix is the logarithm of the unique maximum eigenvalue.

The matrix $\mathcal{L}_{\mathcal{H}}$ can be turned into a Markov matrix of Maximum entropy. For a primitive matrix $M$ with spectral radius $\rho$, and positive right eigenvector $\mathbf{v}$ associated to $\rho$, the stochasticization of $M$ is the following stochastic matrix:

$$
S(M)=\frac{1}{\rho} D^{-1} M D
$$


where $D$ is the diagonal matrix with diagonal entries $D_{i i}=\mathbf{v}_{\mathbf{i}}$. The MEMC transition matrix $P$ and unique stationary probability measure $\pi$ are explicitly given by

$$
P=S\left(\mathcal{L}_{\mathcal{H}}\right) ; \quad \pi_{i}:=\frac{u_{i} v_{i}}{\langle u, v\rangle}, \quad \forall i \in \mathbb{S},
$$

\subsubsection{Finite range Gibbs measures}

For a fixed energy function $\mathcal{H}$ of range $R \geq 2$, there exists an unique stationary Markov measure $\mu$ for which there exist a constant $M>1$ such that [37],

$$
M^{-1} \leq \frac{\mu\left[x_{1, n}\right]}{\exp \left(\sum_{k=1}^{n-R+1} \mathcal{H}\left(x_{k, k+R-1}\right)-(n+R-1) \mathcal{F}[\mathcal{H}]\right)} \leq M,
$$

that attains the supremum (11). The measure $\mu$, as defined by (14), is known in the symbolic dynamics literature as Gibbs measure in the sense of Bowen [38]. All MEMCs belong to this class of measures. Moreover, the classical Gibbs measures in statistical mechanics are particular cases of (14), when $M=1, \mathcal{F}[\mathcal{H}]=\log Z$ and $\mathcal{H}$ is an energy function of range one, leading to an i.i.d stochastic process characterized by the product measure $\mu$. In this case the following holds:

$$
\mu(x)=\frac{e^{\mathcal{H}(x)}}{Z} \quad \forall x \in \mathbb{S} ; \quad Z=\sum_{x \in \mathbb{S}} e^{\mathcal{H}(x)} .
$$

\subsection{Example}

We present here the toy example that we will use to explore statistical properties of spike trains using the non-equilibrium statistical physics approach. We present the transfer matrix technique to compute the Markov transition matrix, its invariant measure and free energy from a potential $\mathcal{H}$.

Consider a range-2 potential with two neurons $(N=2)$. We use the notation introduced in 2.1:

$$
\mathcal{H}\left(\mathbf{x}^{0,1}\right)=h_{1} x_{0}^{1} x_{1}^{2}+h_{2} x_{0}^{2} x_{1}^{1} .
$$

The space state of this problem is given by:

$$
\left(\begin{array}{l}
0 \\
0
\end{array}\right),\left(\begin{array}{l}
0 \\
1
\end{array}\right),\left(\begin{array}{l}
1 \\
0
\end{array}\right),\left(\begin{array}{l}
1 \\
1
\end{array}\right) .
$$

The transfer matrix (12) associated to $\mathcal{H}$ is in this case a $4 \times 4$ matrix

$$
\mathcal{L}_{\mathbf{x} \mathbf{x}^{\prime}}=\left(\begin{array}{cccc}
1 & 1 & 1 & 1 \\
1 & 1 & e^{h_{2}} & e^{h_{2}} \\
1 & e^{h_{1}} & 1 & e^{h_{1}} \\
1 & e^{h_{1}} & 1 & e^{h_{1}+h_{2}}
\end{array}\right)
$$

This matrix obviously satisfies the hypothesis of the Perron-Frobenius theorem. Its unique maximum eigenvalue is

$$
\rho=\frac{1}{2}\left(3+e^{\left(h_{1}+h_{2}\right)}+\sqrt{5+4 e^{h_{1}}+4 e^{h_{2}}+2 e^{\left(h_{1}+h_{2}\right)}+e^{\left(2 h_{1}+2 h_{2}\right)}},\right.
$$

and the free energy

$$
\mathcal{F}[\mathcal{H}]=\log (\rho) .
$$

\section{Statistical properties of Markov maximum entropy measures}

The procedure of finding a maximum entropy model gives us a full statistical model of the system of interest. In this section we discuss the added value that having such a model can provide. 
289

where $\mathbb{E}_{p}\left\{f_{k}\right\}$ is the average with respect to $p$ (maximum entropy measure), which is equal (by assumption) to the average value of $f_{k}$ with respect to the empirical measure from the data $c_{k}$, that constraint of the maximization problem. With equation ((16)) the parameters of the MEMC can be fitted to be consistent with fixed average values of observables.

Suppose we compute from data the average values of the following observables $\left\langle x_{0}^{1} x_{1}^{2}\right\rangle=0.1$ and $\left\langle x_{0}^{2} x_{1}^{1}\right\rangle=0.3$, we solve (16) (two equations and two unknowns) and obtain $h_{1}=-1.98306$ and $h_{2}=1.48406$. With these parameters the following Markov transition matrix and invariant measure are obtained from (13):

$$
P_{\mathbf{x x}^{\prime}}=\left(\begin{array}{cccc}
0.232971 & 0.469441 & 0.0987018 & 0.198886 \\
0.115617 & 0.232971 & 0.216056 & 0.435357 \\
0.549892 & 0.15252 & 0.232971 & 0.0646176 \\
0.272896 & 0.0756914 & 0.509966 & 0.141446
\end{array}\right) \quad \pi(\mathbf{x})=\left(\begin{array}{c}
0.29102 \\
0.248443 \\
0.248443 \\
0.212095
\end{array}\right) .
$$

\subsection{Fluctuation-dissipation relations}

Let $P$ be an ergodic matrix and indexed by the states in some finite set $E$, and $\pi$ be its unique stationary measure. In this general context, for two real-valued function that depend on a fixed finite number of components, we define the $n$-step correlation as

$$
C_{f, g}(n)=\mathbb{E}_{\pi}\left(f\left(X_{0}\right) g\left(X_{n}\right)\right)-\mathbb{E}_{\pi}\left(f\left(X_{0}\right)\right) \mathbb{E}_{\pi}\left(g\left(X_{0}\right)\right) .
$$

In the particular case of MEMC with potentials of range $R>1$ there is a positive time correlation between pairs of observables $f\left(x_{n}\right)$ and $g\left(x_{n+r}\right)$. Suppose the correlations decay fast enough so that (at least)

$$
\sum_{n=0}^{\infty}\left|C_{f, g}(n)\right|<\infty
$$

Then the following sum (known as the Green-Kubo formula [39]) converge and is non-negative:

$$
\sigma_{f_{k}, f_{j}}^{2}=C_{f_{k}, f_{j}}(0)+\sum_{r=1}^{\infty} C_{f_{k}, f_{j}}(r)+\sum_{r=1}^{\infty} C_{f_{j}, f_{k}}(r) .
$$

Additionally, it can be shown that the energy function and the free energy depends smoothly upon maximum entropy parameters. Moreover, the correlations between observables can be obtained from the free energy through:

$$
\sigma_{f_{k}, f_{j}}^{2}=\frac{\partial^{2} \mathcal{F}[\mathcal{H}]}{\partial h_{k} \partial h_{j}}=\frac{\partial \mu\left(f_{j}\right)}{\partial h_{k}} .
$$


The relation between a correlation and a derivative of the free energy is called the fluctuation-dissipation theorem [40]. For a MEMC characterized by $\mu(P, \pi)$, the fluctuation-dissipation relations can be obtained explicitly:

$$
\begin{aligned}
\frac{\partial^{2} \mathcal{F}[\mathcal{H}]}{\partial h_{k} \partial h_{j}}= & \mathbb{E}_{\mu}\left[f_{k} f_{j}\right]-\mathbb{E}_{\mu}\left[f_{k}\right] \mathbb{E}_{\mu}\left[f_{j}\right]+\sum_{r=1}^{\infty} \sum_{\mathbf{x}, \mathbf{x}^{\prime} \in \mathbb{S}}\left(f_{k}(\mathbf{x}) f_{j}\left(\mathbf{x}^{\prime}\right) \pi_{\mathbf{x}} P_{\mathbf{x} \mathbf{x}^{\prime}}^{r}-\mathbb{E}_{\mu}\left[f_{k}\right] \mathbb{E}_{\mu}\left[f_{j}\right]\right) \\
& +\sum_{r=1}^{\infty} \sum_{\mathbf{x}, \mathbf{x}^{\prime} \in \mathbb{S}}\left(f_{j}(\mathbf{x}) f_{k}\left(\mathbf{x}^{\prime}\right) \pi_{\mathbf{x}} P_{\mathbf{x} \mathbf{x}^{\prime}}^{r}-\mathbb{E}_{\mu}\left[f_{k}\right] \mathbb{E}_{\mu}\left[f_{j}\right]\right) .
\end{aligned}
$$

For MEMC built from $K$ observables, the correlations can be conveniently arranged in a $K \times K$ symmetric matrix denoted by $\chi$ (the symmetry refers to the Onsager reciprocity relations [41]).

$$
\chi_{j k}=\frac{\partial^{2} \mathcal{F}[\mathcal{H}]}{\partial h_{k} \partial h_{j}}=\frac{\partial \mu\left(f_{j}\right)}{\partial h_{k}}=\frac{\partial \mu\left(f_{k}\right)}{\partial h_{j}}=\chi_{k j} .
$$

For the example 4.4, we obtain the matrix $\chi_{k j}$ by taking the second derivatives of (15) and evaluate at the parameters found previously,

$$
\chi_{k j}=\left(\begin{array}{cc}
0.0971481 & 0.0606071 \\
0.0606071 & 0.127964
\end{array}\right) .
$$

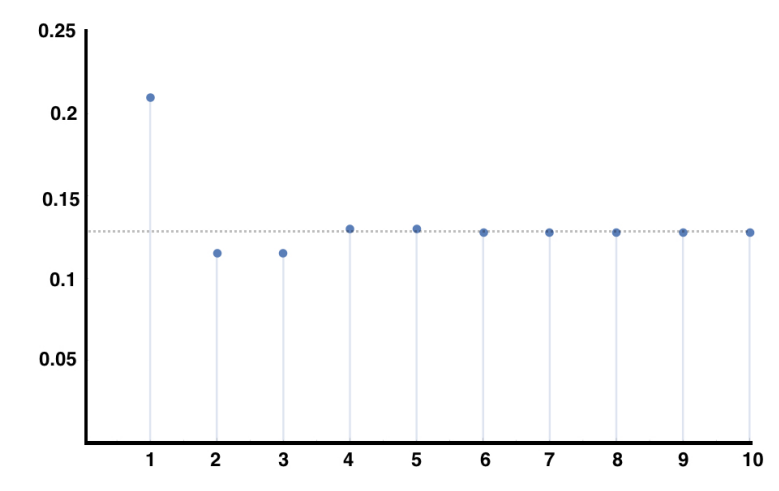

Figure 2. Plot of equation (18) for auto-correlation of the observable $x_{0}^{2} x_{1}^{1}$ with respect to the MEMC consistent with constraints $\left\langle x_{0}^{1} x_{1}^{2}\right\rangle=0.1$ and $\left\langle x_{0}^{2} x_{1}^{1}\right\rangle=0.3$. Here we consider the sum from $r=1$ up to the number in the abscissa. Note the fast convergence towards $\chi_{22}$.

\subsection{Resonances and decay of correlations}

We turn back to the general setting of an arbitrary ergodic matrix $P$ with stationary measure $\pi$ associated to a Markov chain taking values on a finite state space (not necessarily the space of spike-patterns). Without loss of generality assume that $P$ is indexed by the states in $E=\{1,2, \ldots, M\}$. It can be proved that in this case there exists $\left(l_{i}: i=1,2, \ldots, M\right)$ and $\left(r_{i}: i=1,2, \ldots, M\right)$, sets of left and right eigenvectors respectively, associated to the eigenvalues $\left(\rho_{i}: i=1, \ldots, M\right)$. We can assume that the eigenvectors and left and right eigenvalues have been sorted and normalized in such a way that $\rho_{1}=1, l_{1}$ is the unique $P$-stationary probability vector $\pi, r_{1}=(111 \ldots 1)^{T}$, and

$$
\left\langle l_{i} \mid r_{j}\right\rangle=\delta_{i, j}
$$


where $\delta_{i, j}$ is the Kronecker delta, and $\langle u \mid v\rangle=\langle u, v\rangle$ corresponds to the Dirac's bra-ket, $|u\rangle\langle v|=u v^{T}$. With the same notation, the spectral decomposition of $P$ is written:

$$
P=\sum_{i=1}^{M} \rho_{i}\left|r_{i}\right\rangle\left\langle l_{i}\right|
$$

Hence:

$$
P^{n}=\sum_{i=1}^{M} \rho_{i}^{n}\left|r_{i}\right\rangle\left\langle l_{i}\right|
$$

Given two functions $f: E \mapsto \mathbb{R}$ and $g: E \mapsto \mathbb{R}$ the following holds,

$$
\begin{aligned}
C_{f, g}(n) & :=\mathbb{E}_{\pi}\left(f\left(X_{0}\right) g\left(X_{n}\right)\right)-\mathbb{E}_{\pi}\left(f\left(X_{0}\right)\right) \mathbb{E}_{\pi}\left(g\left(X_{0}\right)\right) \\
& =\left\langle\pi \mid f \circ P^{n} g\right\rangle-\langle\pi \mid f\rangle\langle\pi \mid g\rangle .
\end{aligned}
$$

Recall the discussion at previous sections regarding the reverse chain 2.4. Writing $\mathbb{E}_{\pi}^{\overleftarrow{ }}$ for the expectation operator associated to the reverse Markov measure, i.e., to the measure $\mu=\mu(\pi, \overleftarrow{P})$, one can see that

$$
\mathbb{E}_{\pi}\left(f\left(X_{0}\right) g\left(X_{n}\right)\right)=\mathbb{E}_{\pi}^{\leftarrow}\left(f\left(X_{n}\right) g\left(X_{0}\right)\right)
$$

and hence (21) becomes

$$
\left\langle\pi \mid g \circ \overleftarrow{P}^{n} f\right\rangle-\langle\pi \mid f\rangle\langle\pi \mid g\rangle
$$

From (20)

$$
f \circ P^{n} g=\sum_{i=1}^{M}\left\langle l_{i} \mid g\right\rangle\left|f \circ r_{i}\right\rangle
$$

and thus (21) becomes

$$
\begin{aligned}
C_{f, g}(n) & =\sum_{i=1}^{M} \rho_{i}^{n}\left\langle l_{i} \mid g\right\rangle\left\langle\pi \mid f \circ r_{i}\right\rangle-\langle\pi \mid f\rangle\langle\pi \mid g\rangle \\
& =\sum_{i=2}^{M} \rho_{i}^{n}\left\langle l_{i} \mid g\right\rangle\left\langle\pi \mid f \circ r_{i}\right\rangle .
\end{aligned}
$$

We have found an explicit expression for the decay of correlation for observables from the set of eigenvalues and eigenvectors of the transition matrix $P$. This is relevant in the context of spike train statistics because as the matrix $P$ characterizing the spike trains is not expected to be symmetric, its eigenvalues are not necessarily real and modulations in the decay of correlations are expected (resonances). When measuring correlations between observables from data, one may observe this oscillatory situation that resembles resonances, this may be a symptom of a non-equilibrium situation. 


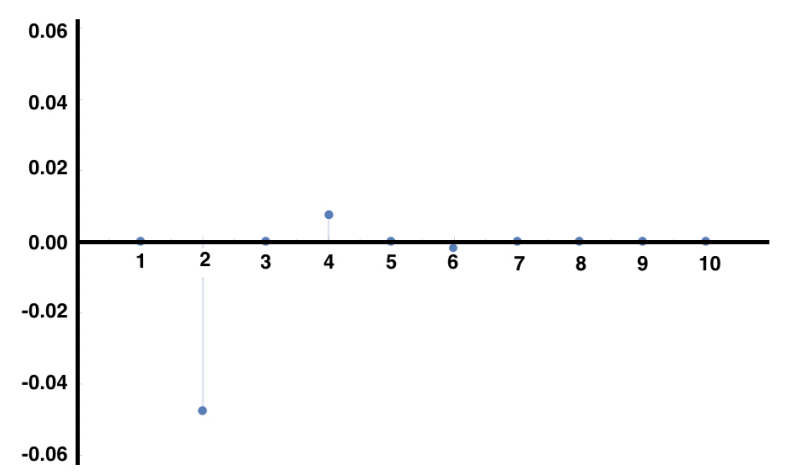

Figure 3. Auto-correlations of the observable $x_{0}^{2} x_{1}^{1}$ for the MEMC with the same parameters as figure 1 . We observe modulations in the decay of correlations due to the complex eigenvalues that arise in the non-symmetric transition matrix which is induced by the irreversibility of the MEMC.

\subsection{Large deviations for average values of observables in MEMC}

Obtaining the probability of "rare" average values of firing rates, pairwise correlations, triplets or non-synchronous observables is relevant in spike train statistics as these observables are likely to play an important role in neuronal information processing, and rare values may convey crucial information or be a symptom that the system in not working properly.

Here, we build from a previous article [42] where it is shown that the SCGF (7) can be obtained directly from the inferred Markov transition matrix $P$ through the Gärtner-Ellis theorem (8). Consider a MEMC with transition matrix $P$. Let $f$ be an observable of finite range and $k \in \mathbb{R}$. We introduce the tilted transition matrix by $f$ of $P$, parametrized by $k$ and denoted by $\widetilde{P}^{(f)}(k)$ [29] as follows:

$$
\widetilde{P}_{i j}^{(f)}(k)=P_{i j} e^{k f(i j)} \quad i, j \in \mathbb{S} .
$$

For MEMC $P$ the tilted transition matrix can be built directly from the spectral properties of the transfer matrix (12) as follows,

$$
\begin{aligned}
\widetilde{P}_{i j}^{(f)}(k) & =\frac{e^{\mathcal{H}_{i j}} v_{j}}{v_{i} \rho} e^{k f(i j)} \\
& =\frac{e^{\left[\mathcal{H}_{i j}+k f(i j)\right]} v_{j}}{v_{i} \rho} \quad i, j \in \mathbb{S} .
\end{aligned}
$$

Recall that $\mathbf{v}$ is the right eigenvector associated to its maximum eigenvalue $\rho$ of the transfer matrix $\mathcal{L}$. Here we also have used the shortcut notation $\mathcal{H}_{i j}$ to indicate that the energy function is built from the elements of the state space $i$ and $j$. Remarkably, this result is valid not only for the observables in the energy function, i.e., from here the LDP of more general observables can be computed.

To obtain an explicit expressions for the SCGF $\lambda_{f}(k)$, is possible to take advantage of the structure of the underlying stochastic process. For instance, for i.i.d. random process $X_{t}$ where $X_{i} \sim X$ from the definition 7 one can obtain that

$$
\lambda(k)=\lim _{t \rightarrow \infty} \frac{1}{t} \ln \mathbb{E}\left[e^{t k A_{t}(f)}\right]^{t}=\ln \mathbb{E}\left[e^{k f(X)}\right],
$$

which is the case of range one observables. Using equation (22), we get that the maximum eigenvalue of the tilted matrix, denoted by $\rho\left(\widetilde{P}_{f}(k)\right)$ is,

$$
\rho\left(\widetilde{P}_{f}(k)\right)=\sum_{j} \pi_{j} e^{k f(j)} \quad j \in \mathbb{S} .
$$


338 Since $\widetilde{P}_{f}$ is a positive matrix the Perron-Frobenius theorem ensures the uniqueness of $\rho\left(\widetilde{P}_{f}(k)\right)$.

For additive observables under the assumption of ergodicity, an straightforward calculation (see for instance [43]) leads us to obtain that

$$
\lambda_{f}(k)=\ln \left(\rho\left(\widetilde{P}^{(f)}\right)\right)
$$

It also can be proved that $\lambda_{f}(k)$, in this case is differentiable [43], setting up the scene to apply the Gärtner-Ellis theorem, which bypasses the direct calculation of $p\left\{A_{T}(f)>s\right\}$ in (6) through $\lambda_{f}(k)$ as its Legendre transform leads to the rate function of $f$ as shown in figure 4 .

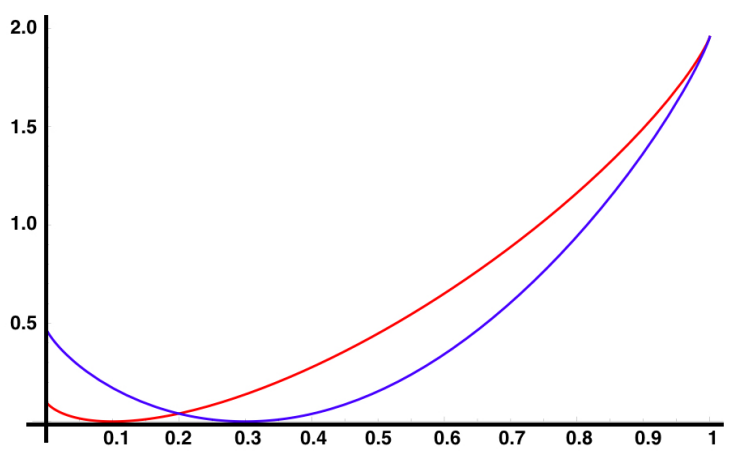

Figure 4. Rate functions of observables $x_{0}^{1} x_{1}^{2}$ in red, and $x_{0}^{2} x_{1}^{1}$ in blue for the MEMC consistent with constraints $\left\langle x_{0}^{1} x_{1}^{2}\right\rangle=0.1$ and $\left\langle x_{0}^{2} x_{1}^{1}\right\rangle=0.3$. The minimum value of both functions coincide with their expected values with respect to the MEMC. Around the minimum Gaussian fluctuations are expected (9). Far from the expected values are the large deviations.

\subsection{Information entropy production}

Given a Markov chain $\left(X_{t}: t \geq 0\right)$ on a general finite state space $E$ with transition matrix $P$ started from the distribution $v$, denote $v^{(n)}$ the distribution of $X_{n}$, namely, for $i \in E$ :

$$
v^{(n)}(i)=\mathbb{P}_{v}\left(X_{n}=i\right)
$$

Obviously, $v^{(0)}=v$, and

$$
v_{j}^{(n+1)}=\sum_{i \in E} v_{i}^{(n)} P_{i j}
$$

The information-theoretic entropy of the probability distribution $v$ at time $n$ is given by

$$
\mathcal{S}_{n}(v):=-\sum_{i \in E} v_{i}^{(n)} \log v_{i}^{(n)},
$$

and the change of entropy over one time step is defined as

$$
\Delta \mathcal{S}_{n}:=\mathcal{S}_{n+1}(v)-\mathcal{S}_{n}(v)
$$

A bit of algebra yields

$$
\Delta \mathcal{S}_{n}=-\sum_{i, j \in E} v_{j}^{(n)} P_{j i} \log \frac{v_{j}^{(n+1)} P_{j i}}{v_{i}^{(n)} P_{i j}}+\frac{1}{2} \sum_{i, j \in E}\left[v_{j}^{(n)} P_{j i}-v_{i}^{(n)} P_{i j}\right] \log \frac{v_{j}^{(n)} P_{j i}}{v_{i}^{(n)} P_{i j}} .
$$


The first term on the R.H.S. above is called information entropy flow and the second term information entropy production [12].

In the stationary case, i.e., when $P$ admits a stationary measure $\pi$ and the chain is started from that distribution, one has that $v^{(n)}=\pi$ for every $n \geq 0$; thus, in this case, the change of entropy rate is zero, i.e., for stationary chains, the information entropy flow equals (minus) information entropy production. This case is the focus on this work. The chain is associated to spike train activity for transitions between $L$-blocks, starting from stationarity we have that the entropy production rate will be explicitly given by

$$
\operatorname{IEP}(P, \pi):=\frac{1}{2} \sum_{\mathbf{x}, \mathbf{x}^{\prime} \in \mathbb{S}^{L}}\left[\pi\left(\mathbf{x}^{\prime}\right) P_{\mathbf{x}^{\prime} \mathbf{x}}-\pi(\mathbf{x}) P_{\mathbf{x} \mathbf{x}^{\prime}}\right] \log \frac{\pi\left(\mathbf{x}^{\prime}\right) P_{\mathbf{x}^{\prime} \mathbf{x}}}{\pi(\mathbf{x}) P_{\mathbf{x} \mathbf{x}^{\prime}}} \geq 0 .
$$

The non-negativity implies that information entropy positive as long as the process violate the detailed balance conditions (4). This is analogous to the second law of thermodynamics [44]. From this equation is easy to realize that if the Markov chain satisfies the detailed balance condition the information entropy production is zero.

At first glance it may seem contradictory the fact that in stationary state the entropy is constant, but at the same time there is a positive "production" of entropy. In stationary state the information entropy production always compensate the information entropy flow, thus the information entropy rate remains constant. In this case we refer to non-equilibrium steady states (NESS).

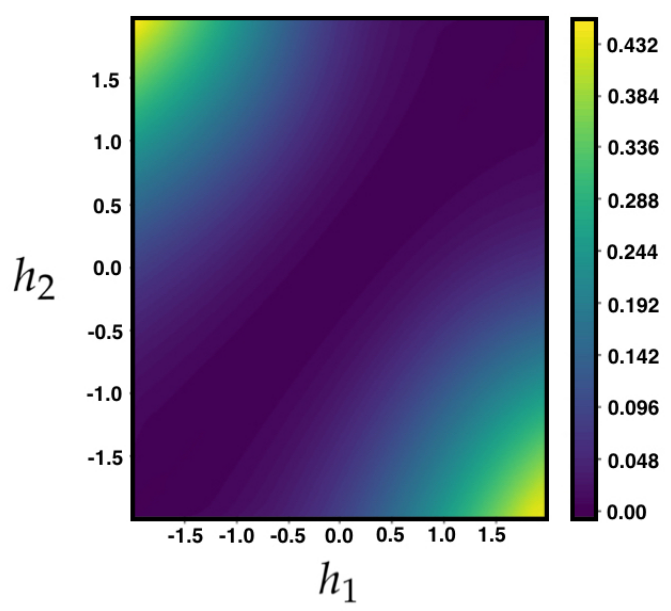

Figure 5. Information entropy production for the MEMC of the example (4.4) for different values of parameters $h_{1}, h_{2}$. Observe that $\operatorname{IEP}(P, \pi)=0$ when $h_{1}=h_{2}$ and that increases as they become more different (more asymmetry in $P$ ).

\subsection{Gallavotti-Cohen fluctuation theorem}

To characterize the fluctuations of the IEP, consider the $\operatorname{MEMC} \mu(P, \pi)$ and the following observable:

$$
W_{n}\left(\mathbf{x}_{0, n}\right)=\frac{1}{n} \ln \left(\frac{\mu\left(\mathbf{x}_{0, n}\right)}{\mu\left(\mathbf{x}_{n, 0}\right)}\right),
$$

It can be shown that $\lim \frac{1}{n} W_{n} \rightarrow \operatorname{IEP}(\pi, P)$. The Gallavotti-Cohen fluctuation theorem is as a statement about properties of the SCGF and rate function of the IEP [14].

$$
\lambda_{W}(k)=\lambda_{W}(-k-1), \quad I_{W}(s)=I_{W}(-s)-s .
$$


This is an universal property of the IEP, i.e., it is independent of the parameters of the MEMC. To compute $\lambda_{W}(k)$ and $I_{W}(s)$, define $A(k)_{i j}=P_{i j}\left[\frac{\pi_{i} P_{i j}}{\pi_{j} P_{j i}}\right]^{k}$. If $\rho(k)$ is the largest eigenvalue of $A(k)$, then $\lim _{n \rightarrow \infty} \ln \mathbb{E}\left(e^{n \lambda W_{n}}\right)=\ln \rho(k)$.

This symmetry holds for a general class of stochastic processes including NESS from Markov chains [45].

These properties have an impact on the large deviations of the time-averaged entropy production rate of the sample trajectory $x_{0, t-1}$ of the Markov chain $p(\pi, P)$ denoted $\frac{W_{t}}{t}$. In our framework, the following relationship always holds,

$$
\frac{p\left\{\frac{W_{t}}{t} \approx s\right\}}{p\left\{\frac{W_{t}}{t} \approx-s\right\}} \asymp e^{t s} .
$$

This means that the positive fluctuations of $\frac{W_{t}}{t}$ are exponentially more probable than negative fluctuations of equal magnitude.
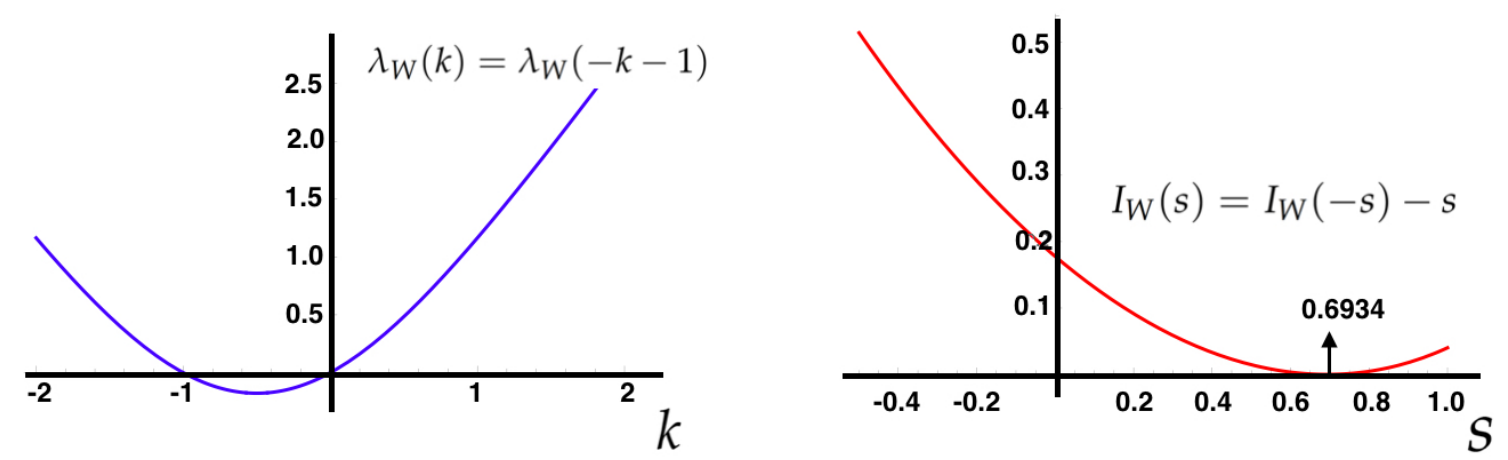

Figure 6. Illustration of the Gallavotti-Cohen symmetry property of the large deviation functions associated to the IEP. Left: SCGF of the IEP of the MEMC with the same parameters considered in the previous examples. Right: Rate function of the observable $W$, the minimum is attained at the expected value of IEP.

\subsection{Linear response}

For a MEMC characterized by $\mu=(P, \pi)$ corresponding to an energy function with fixed parameters $\mathbf{h}$ denoted by $\mathcal{H}_{\mathbf{h}}$, one can obtain the average value of a given observable $f_{k}$ from (16).

Now, consider a perturbed energy denoted by $\tilde{\mathcal{H}}=\mathcal{H}_{\boldsymbol{h}+\delta \boldsymbol{h}}$. Using a Taylor expansion, the average value of an arbitrary observable $f_{k}$ with respect to the MEMC can be obtained $\tilde{\mu}=(\tilde{P}, \tilde{\pi})$ associated to the perturbed energy

The linear response serves to quantify how a small perturbation $\delta \boldsymbol{h}$ of a set of the maximum entropy parameters affects the average values of observables in terms of the unperturbed measure $\mu$. Considering the Taylor expansion of $\mathcal{F}\left[\mathcal{H}_{h+\delta h}\right]$ about $\mathcal{H}_{h}$

$$
\begin{aligned}
\frac{\partial \mathcal{F}\left[\mathcal{H}_{\boldsymbol{h}+\delta \boldsymbol{h}}\right]}{\partial h_{k}} & =\frac{\partial \mathcal{F}\left[\mathcal{H}_{\boldsymbol{h}}\right]}{h_{k}}+\sum_{j} \frac{\partial^{2} \mathcal{F}\left[\mathcal{H}_{h}\right]}{\partial h_{k} h_{j}} \delta h_{j}+O\left(\delta h_{j}\right)^{2}, \\
\mathbb{E}_{\tilde{\mu}}\left[f_{k}\right] & =\mathbb{E}_{\mu}\left[f_{k}\right]+\sum_{j} \frac{\partial^{2} \mathcal{F}\left[\mathcal{H}_{h}\right]}{\partial h_{k} h_{j}} \delta h_{j}+O\left(\delta h_{j}\right)^{2}, \\
\Delta \mathbb{E}[f] & \approx \chi \cdot \delta \mathbf{h} .
\end{aligned}
$$


We use (16) to go from (24) to (25). Observe from (25) that a small perturbation of a parameter $h_{j}$ influences the average value of all other observables in the energy function (as $f_{k}$ is arbitrary). The magnitude of the perturbation is controlled by the second derivatives of the free energy of the unperturbed regime $\mathcal{F}\left[\mathcal{H}_{h}\right]$ (see figure 7 ).
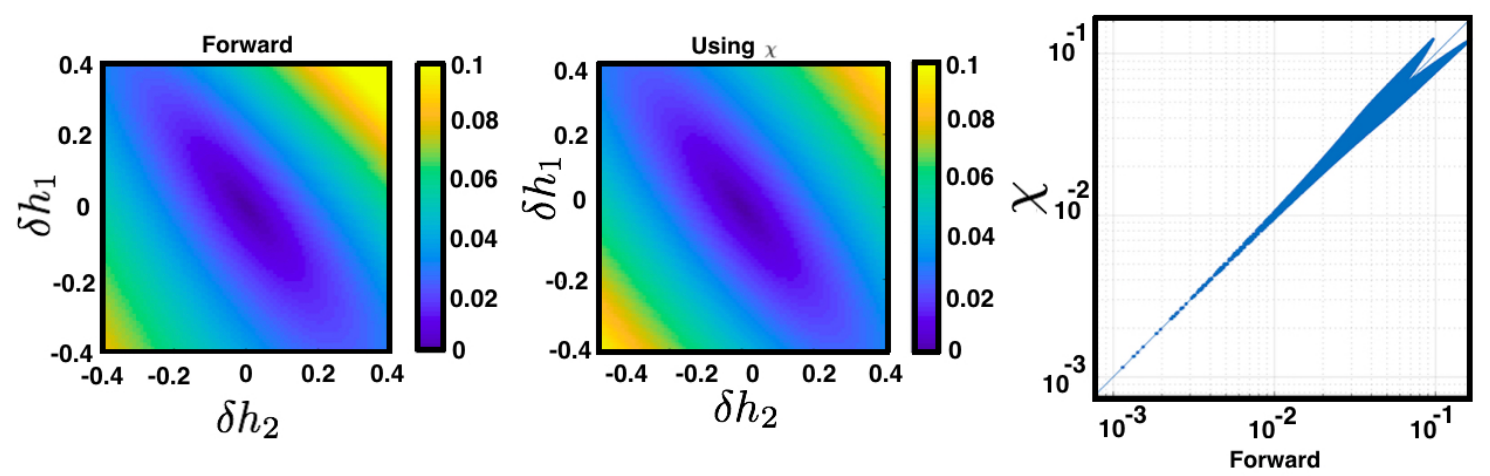

Figure 7. Linear response for the MEMC of the example (4.4) for different values of perturbations $\delta h_{1}$ and $\delta h_{2}$. The colors represent $\left\|\mathbb{E}_{\tilde{u}}\left[f_{k}\right]-\mathbb{E}_{\mu}\left[f_{k}\right]\right\|$ computed using two methods. The "forward" method consist in computing $\mathbb{E}_{\tilde{\mu}}\left[f_{k}\right]$ from $\tilde{\mu}$ and $\mathbb{E}_{\mu}\left[f_{k}\right]$ from $\mu$. The figure in the middle is obtained computing $\left\|\mathbb{E}_{\tilde{\mu}}\left[f_{k}\right]-\mathbb{E}_{\mu}\left[f_{k}\right]\right\|$ from $\chi$ using equation (26). Right) The difference between both methods illustrated in a scatter plot in logarithmic scale.

\section{Discussion and future work}

In this work we explore how one can use maximum entropy methods to capture assymetric temporal aspects of biological processes from experimental data. In particular, we showed how spatio-temporal constraints can produce homogeneous irreducible Markov chains whose unique steady state is in general non-equilibrium (NESS) - thus detailed balance condition is not satisfied causing strictly positive entropy production. This fact highlights that only non-synchronous maximum entropy models induce time irreversible processes, which is one of the key hallmark of biological systems. We have presented a survey of diverse techniques from mathematics and statistical mechanics to study these NESS, which correspond to a rich toolkit that can be employed to study unexplored aspects of spike train statistics. Note that although this article is focused on spike train statistics, the discussed tools and methods can be used in other contexts such as ecology, image processing, and economy, among others.

Non-equilibrium steady states tools and methods may bring new ideas in the field of computational neuroscience. In particular, possible extensions include measuring the entropy production for different choices of spatio-temporal constraints using the maximum entropy method on biological spike train recordings. A more ambitious extension is to explore the relationship between entropy production of given physiological process and relate them to features such as adaptation or learning. Concerning time-dependent neuronal network models, future studies might lead to a better understanding of the impact of particular synaptic topologies of neuronal network models on the corresponding entropy production, decay of correlations, resonances and other sophisticated statistical properties.

Other possible extensions are related to drawbacks of current approaches. This can include limitations of the maximum entropy method related to the requirement of stationarity in the data, which contrast with the fact that information entropy production can still be defined along non-stationary trajectories [14]. Also, another open problem is related to the transfer matrix technique, which currently requires an important computational effort in the case of large neural networks. 
MEMC Maximum entropy Markov chain

SCGF Scaled cumulant generating function

CLT Central limit theorem

${ }_{417}$ LLN Law of large numbers

LDP Large deviation principle

IEP Information entropy production

KSE Kolmogorov-Sinai entropy

NESS Non-equilibrium steady states

${ }_{418}$ Symbol list

$\mathbb{S} \quad\{0,1\}^{N}$ the state space of spike patterns of $N$ neuron

$\Omega \quad$ The set of infinite sequences of spike patterns.

$x_{n}^{k} \quad$ Spiking state of neuron $k$ at time $n$.

$x_{n} \quad$ Spike pattern at time $n$

$x_{t_{1}, t_{2}} \quad$ Spike block from time $t_{1}$ to $t_{2}$.

${ }_{419} \quad v(f) \quad$ Expectation of the observable $f$ w.r.t. the probability measure $v$.

$A_{T}(f) \quad$ Empirical Average value of the observable $f$ considering $T$ spike patterns.

$\mathbb{S}^{R} \quad$ Space of spike blocks of $N$ neurons and length $R$.

$\mathcal{S}[\mu] \quad$ Entropy of the probability measure $\mu$.

$\mathcal{H}$ Energy function.

$\mathcal{F}[\mathcal{H}] \quad$ Free energy. Conflicts of Interest: The authors declare no conflict of interest.
1. Rieke, F.; Warland, D.; de Ruyter van Steveninck, R.; Bialek, W. Spikes, Exploring the Neural Code; M.I.T. 422 Press, 1996.

423 2. Bialek, W. Biophysics: Searching for Principles; Princeton University Press, 2012.

In summary, we believe that these topics are a fertile ground for multi-disciplinary exploration mathematicians, physicists, and neuroscientists. It is our hope that this work borative research among disciplines, which might bring new breakthroughs to . is supported by CONICYT-Beca de Doctorado No. 21170406 Convocatoria 2017. F.R. Chandaria Foundation. R.C. was supported by CONICYT-PAI Inserción 79160120

the main ideas and concepts, wrote and revised the

3. Schneidman, E.; Berry, M.J.; Segev, R.; Bialek, W. Weak pairwise correlations imply strongly correlated network states in a neural population. Nature 2006, 440, 1007-12.

4. Ganmor, E.; Segev, R.; Schneidman, E. Sparse low-order interaction network underlies a highly correlated and learnable neural population code. Proceedings of the National Academy of Sciences of the United States of America 2011, 108, 9679-84.

5. Tkacik, G.; Marre, O.; Amodei, D.; Schneidman, E.; Bialek, W.; Berry, M.J. Searching for collective behavior in a large network of sensory neurons. PLoS computational biology 2014, 10, e1003408.

6. Palsso, B. Systems Biology: Properties of Reconstructed Networks; Cambridge University Press, 2006.

7. Tang, A.; Jackson, D.; Hobbs, J.; Chen, W.; Smith, J.; Patel, H.; Prieto, A.; Petrusca, D.; Grivich, M.; Sher, A.; Hottowy, P.; W.Dabrowski.; Litke, A.; Beggs, J. A Maximum Entropy Model Applied to Spatial and Temporal Correlations from Cortical Networks In Vitro. The Journal of Neuroscience 2008, 28, 505-518. 
8. Marre, O.; El Boustani, S.; Frégnac, Y.; Destexhe, A. Prediction of spatiotemporal patterns of neural activity from pairwise correlations. Physical review letters 2009, 102.

9. Vasquez, J.; Palacios, A.; Marre, O.; Berry II, M.; Cessac, B. Gibbs distribution analysis of temporal correlation structure on multicell spike trains from retina ganglion cells. J. Physiol. Paris 2012, 106, 120-127.

10. Mora, T.; Deny, S.; Marre, O. Dynamical criticality in the collective activity of a population of retinal neurons. Phys. Rev. Lett. 2015, 115.

11. Cofré, R.; Cessac, B. Exact computation of the maximum entropy potential of spiking neural networks models. Physical Review E 2014, 107, 368-368.

12. Cofré, R.; Maldonado, C. Information Entropy Production of Maximum Entropy Markov Chains from Spike Trains. Entropy 2018, 20.

13. Schulman, L.S. Time's arrows and quantum measurement; Cambridge University Press, 1997.

14. Jiang, D.Q.; Qian, M.; Qian, M.P. Mathematical Theory of Non-equilibrium Steady States; Springer, 2004.

15. Schrödinger, E. What Is Life? The Physical Aspect of the Living Cell; Cambridge University Press, 1944.

16. Deem, M. Mathematical adventures in biology. Phys Today 2007, 60, 42-47.

17. Prigogine, I. Nonequilibrium Statistical Mechanics; Monographs in Statistical Physics, Interscience publishers, John Wiley \& Sons, 1962.

18. Filyukov, A.; Karpov, V. Description of steady transport processes by the method of the most probable path of evolution. Inzhenerno-Fizicheskii Zhurnal 1967, 13, 624-630.

19. Filyukov, A.; Karpov, V. Method of the most probable path of evolution in the theory of stationary irreversible processes. Inzhenerno-Fizicheskii Zhurnal 1967, 13, 798-804.

20. Favretti, M. The Maximum Entropy Rate Description of a Thermodynamic System in a Stationary Non-Equilibrium State. Entropy 2009, 4, 675-687.

21. Monthus, C. Non-equilibrium steady states: maximization of the Shannon entropy associated with the distribution of dynamical trajectories in the presence of constraints. J Stat Mech: Theor Exp. 2011, 3, P03008.

22. Shi, P.; Qian, H., Frontiers in Computational and Systems Biology, J. Feng, W. Fu and F. Sun Eds; Springer, 2010; chapter Irreversible Stochastic Processes, Coupled Diffusions and Systems Biochemistry., pp. 175-201.

23. Galves, A.; Löcherbach, E. Infinite Systems of Interacting Chains with Memory of Variable Length-A Stochastic Model for Biological Neural Nets. Journal of Statistical Physics 2013, 151, 896-921.

24. Cofré, R.; Cessac, B. Dynamics and spike trains statistics in conductance-based Integrate-and-Fire neural networks with chemical and electric synapses. Chaos, Solitons and Fractals 2013, 50, 13-31.

25. Halmos, P.R. Measure theory; Graduate Texts in Mathematics. New York: Springer-Verlag, 1974.

26. Levin, D.; Peres, Y. Markov Chains and Mixing Times 2nd. ed.; American Mathematical Society, 2017.

27. Jones, G.L. On the Markov chain central limit theorem. Probab. Surv. 2004, 1, 299-320.

28. Ellis, R. Entropy, Large deviations and Statistical Mechanics; Springer, Berlin, 1985.

29. Touchette, H. A Basic Introduction to Large Deviations: Theory, Applications, Simulations. http://arxiv.org/pdf/1106.4146v3.pdf 2012.

30. Dembo, A.; Zeitouni, O. Large deviations techniques and applications; Vol. 38, Stochastic Modelling and Applied Probability, Springer-Verlag, Berlin, 2010.

31. Touchette, H. The large deviation approach to statistical mechanics. Phys. Rep. 2009, 1-3, 1-69.

32. Ruelle, D. Thermodynamic formalism; Addison-Wesley,Reading, Massachusetts, 1978.

33. Jaynes, E. Information theory and statistical mechanics. Phys. Rev. 1957, 106.

34. Marre, O.; El Boustani, S.; Frégnac, Y.; Destexhe, A. Prediction of spatiotemporal patterns of neural activity from pairwise correlations. Physical review letters 2009, 102.

35. Tkacik, G.; Marre, O.; Mora, T.; Amodei, D.; Berry II, M.J.; Bialek, W.; Tkačik, G.; Marre, O.; Mora, T.; Amodei, D.; Berry II, M.J.; Bialek, W. The simplest maximum entropy model for collective behavior in a neural network. Journal of Statistical Mechanics: Theory and Experiment 2013, 2013, P03011.

36. Seneta, E. Non-negative Matrices and Markov Chains; Springer, 2006.

37. Bowen, R. Equilibrium states and the ergodic theory of Anosov diffeomorphisms. Second revised version.; Vol. 470, Lect. Notes.in Math., Springer-Verlag, 2008.

38. Chazottes, J., Nonlinear Dynamics New Directions; Springer, 2015; chapter Fluctuations of observables in dynamical systems: from limit theorems to concentration inequalities. González-Aguilar H., Ugalde E. Eds, pp. 47-85.

39. Gaspard, P. Chaos, scattering and statistical mechanics; Vol. 9, Cambridge Non-Linear Science series, 1998. 
488 40. Bettolo, U.M.; Puglisi, A.; Rondoni, L.; Vulpiani, A. Fluctuation-dissipation: Response theory in statistical $489 \quad$ physics. Physics Reports 2008, 461, 111-195.

490 41. Gaspard, P. Random paths and current fluctuations in nonequilibrium statistical mechanics. Journal of $491 \quad$ Mathematical Physics 2014, 55.

492 42. Cofré, R.; Maldonado, C.; Rosas, F. Large Deviations Properties of Maximum Entropy Markov Chains $493 \quad$ from Spike Trains. Entropy 2018, 20.

494 43. Ellis, R.S. The theory of large deviations and applications to statistical mechanics. In Long-range interacting 495 systems; Oxford Univ. Press, 2010.

496 44. Nicolis, G.; Nicolis, C. Foundations of Complex Systems: Emergence, Information and Prediction; World Scientific, 4972012

498 45. Maes, C. The fluctuation theorem as a Gibbs property. J. Stat. Phys. 1999, 95, 367-392. 